Pacific Journal of Mathematics

ELLIPTIC SURFACES WITH AN AMPLE DIVISOR OF GENUS 


\title{
ELLIPTIC SURFACES WITH AN AMPLE DIVISOR OF GENUS TWO
}

\author{
Fernando Serrano
}

\begin{abstract}
Beltrametti, Lanteri and Palleschi have recently started the classification of smooth algebraic surfaces having an ample divisor of arithmetic genus two (Arkiv för Mat. 25 (1987), 189-210). Their results for the class of elliptic surfaces can be considerably improved. The present paper focuses on elliptic surfaces $S$ with Kodaira dimension one, $\chi \mathscr{O}_{S}=0$, and such that the (unique) elliptic fibration has a rational base. The result is the following: if $S$ contains a genus two ample divisor then $S$ is of the form $S=(D \times E) / G$ where $G$ is a group acting on two curves $D$ and $E, E$ is elliptic, $G$ is either $\mathbb{Z}_{2} \times \mathbb{Z}_{2}, \mathbb{Z}_{2} \times \mathbb{Z}_{6}$ or $\mathbb{Z}_{4} \times \mathbb{Z}_{4}$ and $D$ has genus 2,2 and 3 respectively. Moreover, the existence of such polarized surfaces is shown by a concrete example.
\end{abstract}

0. Introduction. The classification of smooth projective surfaces by means of numerical invariants (degree or genus) of their hyperplane sections has been a recurring theme in the literature for quite a long time. Recently, the subject has received a renewed impulse by relaxing the hypothesis and considering data from ample divisors as well. Within this general program one can place the work of Beltrametti, Lanteri and Palleschi [3] about smooth surfaces containing an ample divisor of genus 2, subsequently generalized by Beltrametti and Sommese to include singular surfaces as well ([4]). Fujita has studied the higher dimensional situation in [6], [7].

The present paper is a contribution to the understanding of smooth elliptic surfaces endowed with an ample divisor of genus 2, possibly non-effective. Here we shall complete the classification for a particular class of surfaces. More precisely, our set-up is the following: $S$ will be an elliptic surface of Kodaira dimension 1 and $\chi \mathscr{O}_{S}=0$, which contains an ample divisor of genus 2 , and such that the (unique) elliptic fibration on $S$ has a rational base. (See Theorem 2.1 to put these surfaces within a more general framework.) Under these hypotheses we shall prove: there exists a finite group $G$ acting faithfully on a curve $D$ and on an elliptic curve $E$ such that $D / G \simeq \mathbb{P}^{1}, E / G$ is elliptic, $S \simeq(D \times E) / G$ (where $G$ is acting on $D \times E$ componentwise) 
and only the following cases can occur:

\begin{tabular}{|c|c|c|}
\hline genus of $D$ & $G$ & $\begin{array}{c}\text { multiplicities of the singular } \\
\text { fibres of the elliptic fibration }\end{array}$ \\
\hline 2 & $\mathbb{Z}_{2} \times \mathbb{Z}_{2}$ & $(2,2,2,2,2)$ \\
2 & $\mathbb{Z}_{2} \times \mathbb{Z}_{6}$ & $(2,6,6)$ \\
3 & $\mathbb{Z}_{4} \times \mathbb{Z}_{4}$ & $(4,4,4)$ \\
\hline
\end{tabular}

The proof is based on a structure theorem for the surfaces considered (Theorem 1.2) which reduces the problem to the study of abelian Galois coverings of curves. Combining this information with the results of [3] yields the three cases of the table above.

In [3], the question on whether there actually existed properly elliptic surfaces having a genus 2 ample divisor was left unanswered. Guided by the explicitness of our result we are going to construct such an example. This will be done in the last part of the paper.

1. Notation and preliminaries. We will be working over the field of complex numbers. A surface (respectively, a curve) is a smooth connected algebraic variety of dimension 2 (respectively, 1). Given a divisor $D$ on a surface $S$, we denote by $\mathscr{O}_{S}(D)$ the associated invertible sheaf, and $\chi \mathscr{O}_{S}(D):=\sum_{i=0}^{2}(-1)^{i} \operatorname{dim} H^{i} \mathscr{O}_{S}(D)$. The irregularity and geometric genus of $S$ are defined as $q(S):=\operatorname{dim} H^{1} \mathscr{O}_{S}$, $p_{g}(S):=\operatorname{dim} H^{2} \mathscr{O}_{S}$ respectively. $K_{S}$ always stands for the canonical divisor of $S$, and $g(C)$ for the (arithmetic) genus of a curve or divisor $C$. The notation $D \equiv D^{\prime}$ means that the divisors $D, D^{\prime}$ are numerically equivalent. The free abelian group of divisors modulo numerical equivalence is denoted $\operatorname{Num}(S)$. The cohomology of the exponential sequence exhibits $\operatorname{Num}(S)$ as a subgroup of $H^{2}(S, \mathbb{Z}) /$ (torsion), and both groups coincide provided that $p_{g}(S)=0$. The set of integers $\mathbb{Z}$ modulo $(d) \mathbb{Z}$ is denoted $\mathbb{Z}_{d}$.

An elliptic fibration is a morphism $\varphi: S \rightarrow C$ from a surface onto a curve, whose general fibre is an elliptic curve. The fibration is said to be relatively minimal if no fibre contains a $(-1)$-curve. Let $F$ be any fibre of $\varphi$. The multiplicity of $F$ is defined to be the greatest common divisor of the multiplicities of its irreducible components. Multiple fibres are the ones with multiplicity greater than one. 
Let $B$ be a curve and $G$ a finite group acting on $B$. Write $C:=$ $B / G$, and let $h: B \rightarrow C$ be the natural projection. If $h$ is ramified at $P \in B$, then $P$ is called a ramification point, and $h(P) \in C$ is a branch point. Inasmuch as $h$ is a Galois map, one sees that all points in the same fibre of $h$ have equal multiplicity. Thus we can speak of the multiplicity or branching order of a branch point. In particular, if $Q_{1}, \ldots, Q_{t} \in C$ are the branch points of $h$ with multiplicities $m_{1}, \ldots, m_{t}$, and $k:=$ order of $G$, then Hurwitz's formula yields

$$
2 g(B)-2=k(2 g(C)-2)+\sum_{i=1}^{t}\left(k / m_{i}\right)\left(m_{i}-1\right) \text {. }
$$

Furthermore, the set of elements in $G$ fixing a given point of $B$ forms a cyclic group.

LEMMA 1.1. Let $\varphi: S \rightarrow C$ be a relatively minimal elliptic fibration. Then $\chi \mathscr{O}_{S}=0$ if and only if all singular fibres of $\varphi$ are multiples of smooth elliptic curves. In this case, all smooth fibres are isomorphic to each other.

Proof. In view of Theorems 6 and 7 of Chapter IV in [14], the topological Euler characteristic $e(S)$ of $S$ vanishes if and only if all singular fibres of $\varphi$ are multiples of smooth elliptic curves. Noether's formula $12 \chi \mathscr{O}_{S}=e(S)+K_{S}^{2}$ combined with $K_{S}^{2}=0$ shows that $\chi \mathscr{O}_{S}=0$ is equivalent to $e(S)=0$. As for the statement that all smooth fibres are isomorphic, see ([2], VI. 7 and 8).

For the surfaces we are interested in there is a very complete structure theorem:

THeOREM 1.2. Let $\varphi: S \rightarrow C$ be a relatively minimal elliptic fibration with $\chi \mathscr{O}_{S}=0$ and $q(S)=g(C)+1$. Then there exist smooth curves $D$ and $E$, with $E$ elliptic, and an abelian group $G:=\mathbb{Z}_{a} \times \mathbb{Z}_{b}$ acting faithfully on $D$ and $E$, so that $G$ acts on $E$ by translations, $S \simeq(D \times E) / G$ (where $G$ is acting on $D \times E$ componentwise), $C \simeq$ $D / G$ and, with these identifications, $\varphi$ coincides with the natural projection $(D \times E) / G \rightarrow(D / G)$.

Proof. Having into account Lemma 1.1 one can proceed as in Chapter VI of [2]. For the details and a more general setting we refer to [13]. The structure of $G$ will follow from the considerations below. 
Künneth's formula yields

$$
\begin{aligned}
H_{1}((D \times E) / G ; \mathbb{Q}) & \simeq H_{1}(D \times E, \mathbb{Q})^{G} \simeq H_{1}(D, \mathbb{Q})^{G} \times H_{1}(E, \mathbb{Q})^{G} \\
& =H_{1}(D / G, \mathbb{Q}) \times H_{1}(E / G, \mathbb{Q}) .
\end{aligned}
$$

It follows that $q(S)=g(D / G)+g(E / G)$. The hypotheses imply that $g(E / G)=1$, i.e., $G$ acts on $E$ by translations. In particular, $G \simeq \mathbb{Z}_{a} \times \mathbb{Z}_{b}$ for some $a, b \in \mathbb{Z}$, as desired.

Let $S=(D \times E) / G$ be a surface as in Theorem 1.2. The fact that $E \rightarrow(E / G)$ is étale implies that all fibres of the natural projection $S \rightarrow(E / G)$ are smooth (and isomorphic to $D)$. Let $P \in(D / G)$ be a point whose branching order by the projection $D \rightarrow(D / G)$ is $m$. Then the fibre of $\varphi: S \rightarrow(D / G)$ over $P$ has multiplicity $m$ and is of the form $m B$, with $B$ being a smooth elliptic curve. All smooth fibres of $\varphi$ are isomorphic to $E$.

Under the hypothesis of Theorem 1.2 the multiplicities of the fibres of $\varphi$ enjoy a peculiar property, which is shown in the following proposition. One proof was given by Katsura and Ueno in ([10], Cor. 4.1). Actually they only dealt with the case $C=\mathbb{P}^{1}$, but their method works for an arbitrary $C$ as well. A more structural proof can be found in [13], which shows that the phenomenon stems from the commutativity of the group $G$ in Theorem 1.2.

Proposition 1.3. Let $\varphi: S \rightarrow C$ be a relatively minimal elliptic fibration with $\chi \mathscr{O}_{S}=0$ and $q(S)=g(C)+1$. By $m_{1}, \ldots, m_{t}$ we denote the multiplicities of the singular fibres of $\varphi$. Then either $t=0$ or $t \geq 2$ and for all $i=1, \ldots, t$, the integer $m_{i}$ divides the least common multiple of $\left\{m_{1}, \ldots, m_{i-1}, m_{i+1}, \ldots, m_{t}\right\} \quad\left(m_{i}\right.$ deleted $)$.

We will also need the following result. An alternate proof is found in [12].

Proposition 1.4. Keep the hypothesis of the previous proposition, and denote by $\mu$ the least common multiple of $m_{1}, \ldots, m_{t}$. Let $E$ be a general fibre of $\varphi$, and $r \in \mathbb{Q}$ any number. Then $r E \in \operatorname{Num}(S)$ if and only if $r \mu \in \mathbb{Z}$.

Proof. Let us first see that $(1 / \mu) E \in \operatorname{Num}(S)$. Since $\mu / m_{1}, \ldots$, $\mu / m_{t}$ are relatively prime, there exist integers $\lambda_{1}, \ldots, \lambda_{t}$ such that $\sum_{i=1}^{t} \lambda_{i}\left(\mu / m_{i}\right)=1$. Let $m_{1} B_{1}, \ldots, m_{t} B_{t}$ stand for the multiple fibres of $\varphi$. We have $B_{i} \equiv\left(1 / m_{i}\right) E$, and thus $(1 / \mu) E \equiv \sum_{i=1}^{t} \lambda_{i} B_{i} \in$ $\operatorname{Num}(S)$, as we claimed. 
Now suppose $r E \in \operatorname{Num}(S)$ with $r \mu \notin \mathbb{Z}$, and choose a divisor $L$ in the numerical equivalence class $r E$. The canonical bundle formula says $K_{S}=\varphi^{*}(D)+\sum_{i=1}^{t}\left(m_{i}-1\right) B_{i}$ for some divisor $D$ on $C$ ([1]). In particular $K_{S} \equiv(d / \mu) E, d \in \mathbb{Z}$. Now we claim that $L$ is non-effective. Otherwise, since $L E=0$ it would follow that $L$ is a combination of components of some fibres. By Lemma 1.1 all fibres of $\varphi$ are irreducible, even if non-reduced. Hence we get a contradiction with the assumption $r \mu \notin \mathbb{Z}$. The same arguments yield the vanishing of $H^{0} \mathscr{O}_{S}(K-L), H^{0} \mathscr{O}_{S}(L-E)$ and $H^{0} \mathscr{O}_{S}(K-L+E)$. Consider the exact sequence

$$
0 \rightarrow \mathscr{O}_{S}(L-E) \rightarrow \mathscr{O}_{S}(L) \rightarrow \mathscr{O}_{E}(L) \rightarrow 0 .
$$

From Riemann-Roch $\chi \mathscr{O}_{S}(L-E)=0$, and combining with the preceding assertions it follows that $H^{1} \mathscr{O}_{S}(L-E)=0$. The above sequence implies $H^{0} \mathscr{O}_{E}(L)=0$. On the other hand, we may observe that the Albanese map of $S$ is not a composite of $\varphi$ (e.g. [2], V.15). In particular $\operatorname{Alb}(E) \rightarrow \operatorname{Alb}(S)$ is a non-trivial morphism. Inasmuch as Picard and Albanese varieties are dual to one another, we deduce that $\operatorname{Pic}^{0}(S) \rightarrow \operatorname{Pic}^{0}(E) \simeq E$ is non-trivial, hence surjective. Now we may choose $\delta \in \operatorname{Pic}^{0}(S)$ such that $\left.\delta\right|_{E}=\left.L\right|_{E}$. The cohomology sequence of

$$
0 \rightarrow \mathscr{O}_{S}(L-\delta-E) \rightarrow \mathscr{O}_{S}(L-\delta) \rightarrow \mathscr{O}_{E} \rightarrow 0
$$

shows that either $H^{0} \mathscr{O}_{S}(L-\delta) \neq 0$ or $H^{1} \mathscr{O}_{S}(L-\delta-E) \neq 0$. But the considerations above yield a contradiction since $L-\delta \equiv L$.

REMARK 1.5. If $L$ is a divisor on $S$ and $r$ is a rational number we have that $r L \in \operatorname{Num}(S)$ if and only if $r L \in H^{2}(S, \mathbb{Z}) /($ torsion). This is a consequence of the exact sequence

$$
H^{1} \mathscr{O}_{S} \rightarrow H^{1} \mathscr{O}_{S}^{*} \rightarrow H^{2}(S, \mathbb{Z}) \rightarrow H^{2} \mathscr{O}_{S},
$$

derived from the exponential sequence. Notice that the torsion of $H^{2}(S, \mathbb{Z})$ is algebraic, that is, lies in the image of $H^{1} \mathscr{O}_{S}^{*} \simeq \operatorname{Pic} S$.

2. Elliptic surfaces with a genus 2 ample divisor. Beltrametti, Lanteri and Palleschi have set out in [3] to classify surfaces having a genus 2 ample divisor. Here we will complete the classification for a particular kind of elliptic surfaces of Kodaira dimension 1. We are going to retake their work at the point they left it in loc. cit. On the one hand, Proposition 1.3 above rules out most cases in the table on ([3], p. 198). Moreover, we must add a missing case in their list: elliptic fibrations over an elliptic curve having as only singular fibre a double curve are still possible. (The argument in step b), p. 195 of [3] is 
incomplete.) Adding these two remarks to the results in [3] we can exhibit the updated list as follows:

THeOREM 2.1. Let $S$ be a smooth surface of Kodaira dimension 1 which contains an ample divisor $L$ of genus 2 . Denote by $\varphi: S \rightarrow$ $C$ the (unique) elliptic fibration on $S$, and let $\left\{m_{1}, \ldots, m_{t}\right\}$ be the multiplicities of the multiple fibres of $\varphi$. Then $S$ is a minimal surface with $p_{g}(S)=0, L^{2}=L K_{S}=1$, and the only possibilities are shown in the table below:

\begin{tabular}{|c|c|c|c|}
\hline Type & $q(S)$ & $g(C)$ & $\left(m_{1}, \ldots, m_{t}\right)$ \\
\hline 1 & 1 & 0 & $(2,2,2,2,2)$ \\
2 & 1 & 0 & $(4,4,4)$ \\
3 & 1 & 0 & $(2,6,6)$ \\
4 & 1 & 1 & $(2)$ \\
5 & 0 & 0 & $(2,2,2)$ \\
6 & 0 & 0 & $(3,3)$ \\
7 & 0 & 0 & $(2,4)$ \\
8 & 0 & 0 & $(2,3)$ \\
\hline
\end{tabular}

We aim at describing more in detail the first three cases of the preceding list. The final result will be:

THeOREM 2.2. Let $S$ be a surface satisfying the hypothesis of Theorem 2.1 and belonging to Types 1, 2 or 3 of the table above. Then there exists a group $G$ acting faithfully on two smooth curves $D, E$ such that :

(i) $D / G \simeq \mathbb{P}^{1}$ and $g(E)=g(E / G)=1$. Hence $G$ is acting on $E$ by translations.

(ii) $S \simeq(D \times E) / G$, where $G$ is acting on $D \times E$ componentwise, and the elliptic fibration $\varphi: S \rightarrow \mathbb{P}^{1}$ is the natural projection $(D \times$ $E) / G \rightarrow(D / G)$.

(iii) Let $D_{0}$ (respectively $E_{0}$ ) denote a general fibre of the natural map $(D \times E) / G \rightarrow(E / G)$ (resp. of $(D \times E) / G \rightarrow(D / G))$, and set $g:=g(D)=g\left(D_{0}\right), k:=$ order of $G$. Then

$$
L \equiv(1 /(2 g-2)) D_{0}+((g-1) / k) E_{0} \text {. }
$$


(iv) Only the following cases can occur:

\begin{tabular}{|c|c|c|}
\hline$g$ & $G$ & $\left(m_{1}, \ldots, m_{t}\right)$ \\
\hline 2 & $\mathbb{Z}_{2} \times \mathbb{Z}_{2}$ & $(2,2,2,2,2)$ \\
3 & $\mathbb{Z}_{4} \times \mathbb{Z}_{4}$ & $(4,4,4)$ \\
2 & $\mathbb{Z}_{2} \times \mathbb{Z}_{6}$ & $(2,6,6)$ \\
\hline
\end{tabular}

In fact, the two projections $S \rightarrow(E / G), S \rightarrow(D / G)$ are the only fibrations on $S$, because $\left\{E_{0}, D_{0}\right\}$ form a basis of $H^{2}(S, \mathbb{Z}) /($ torsion $)$.

Before proceeding with the proof of this theorem we shall state three auxiliary results.

Proposition 2.3 ([9]). Every automorphism of a curve of genus $g \geq$ 2 has order not greater than $2(2 g+1)$.

Proposition 2.4 ([11]). Let $G=\mathbb{Z}_{a} \times \mathbb{Z}_{b}$ be a non-cyclic abelian group with a dividing $b$, and suppose that $G$ is acting faithfully on $a$ curve of genus $g \geq 2$. Then $g$ is bounded below as follows:

\begin{tabular}{|c|c|}
\hline$G$ & lower bound for $g$ \\
\hline $\mathbb{Z}_{2} \times \mathbb{Z}_{2}$ & 2 \\
$\mathbb{Z}_{2} \times \mathbb{Z}_{4}$ & 3 \\
$\mathbb{Z}_{3} \times \mathbb{Z}_{3}$ & 4 \\
otherwise & $1+(a b / 2)(1-1 / a-2 / b)$ \\
\hline
\end{tabular}

Proposition 2.5. Let $H$ be a finite abelian group of automorphisms of $\mathbb{P}^{1}$, and denote by $\left(d_{1}, \ldots, d_{r}\right)$ the multiplicities of the branch points of $f: \mathbb{P}^{1} \rightarrow\left(\mathbb{P}^{1} / H\right) \simeq \mathbb{P}^{1}$. Then the possibilities are:

\begin{tabular}{|c|c|}
\hline$H$ & $\left(d_{1}, \ldots, d_{r}\right)$ \\
\hline $\mathbb{Z}_{n}$ & $(n, n)$ \\
$\mathbb{Z}_{2} \times \mathbb{Z}_{2}$ & $(2,2,2)$ \\
\hline
\end{tabular}


Sketch of proof. This is a very classical result. For $H$ any finite group acting on $\mathbb{P}^{1}$, Hurwitz's formula yields the possibilities for $\left(d_{1}, \ldots, d_{r}\right)$ and for the order of $H$ (e.g. [5], p. 209). Let $B$ denote the branch locus of $f$. The fundamental group of the topological covering space $\mathbb{P}^{1}-f^{-1}(B) \rightarrow \mathbb{P}^{1}-B$ sits in an exact sequence

$$
\pi_{1}\left(\mathbb{P}^{1}-f^{-1}(B)\right) \rightarrow \pi_{1}\left(\mathbb{P}^{1}-B\right) \rightarrow H \rightarrow 0
$$

which, together with the ramification data, yields a presentation of $H$. In general, $H$ will be one of the following groups: $\mathbb{Z}_{n}, D_{n}$ (dihedral), $A_{4}, A_{5}$ (alternating), $S_{4}$ (symmetric). Only $\mathbb{Z}_{n}$ and $D_{2}$ are abelian.

Proof of Theorem 2.2. Parts (i) and (ii) of the statement are immediate consequences of Theorem 1.2. Write $G:=\mathbb{Z}_{a} \times \mathbb{Z}_{b}$ with $a$ dividing $b$, so that $k=a b$. Obviously, $D_{0}^{2}=E_{0}^{2}=0, D_{0} E_{0}=k$. Inasmuch as $S$ is a minimal elliptic surface one has $K_{S} \equiv d E_{0}$, $d \in \mathbb{Q}$. But the adjunction formula yields $2 g-2=D_{0} K_{S}=d k$, and thus $K_{S} \equiv((2 g-2) / k) E_{0}$. One knows that $g \geq 2$ because $S$ is of Kodaira dimension 1 . On the other hand, by Künneth's formula it follows that

$$
\begin{aligned}
H^{2}(S, \mathbb{Q}) & =H^{2}(D \times E, \mathbb{Q})^{G}=\bigoplus_{i=1}^{2}\left[H^{i}(D, \mathbb{Q})^{G} \otimes H^{2-i}(E, \mathbb{Q})^{G}\right] \\
& =\bigoplus_{i=0}^{2}\left[H^{i}(D / G, \mathbb{Q}) \otimes H^{2-i}(E / G, \mathbb{Q})\right] .
\end{aligned}
$$

One concludes that $H^{2}(S, \mathbb{Q})$ is 2-dimensional, and $D_{0}, E_{0}$ form a basis. Thus one can write $L \equiv \alpha D_{0}+\beta E_{0}$ for some $\alpha, \beta \in \mathbb{Q}$. Now part (iii) of our theorem follows by taking into account $L^{2}=L K_{S}=1$ (Theorem 2.1). At this point we begin the analysis of the group $G$. Put $N:=-2+\sum_{i=1}^{t}\left(1-1 / m_{i}\right)$. By applying Hurwitz's formula to the ramified covering $D \rightarrow(D / G) \simeq \mathbb{P}^{1}$ one obtains $k=(2 g-2) / N$. Therefore we get the following table:

\begin{tabular}{|c|c|c|}
\hline Type & $\left(m_{1}, \ldots, m_{t}\right)$ & $k$ \\
\hline 1 & $(2,2,2,2,2)$ & $4(g-1)$ \\
2 & $(4,4,4)$ & $8(g-1)$ \\
3 & $(2,6,6)$ & $12(g-1)$ \\
\hline
\end{tabular}


If $G$ is cyclic then Proposition 2.3 yields that either $S$ belongs to type 1 or $g=2$ and $S$ is of Type 2. We will see later that neither case occurs. Suppose now that $G$ is non-cyclic, and recall Proposition 2.4. $\mathbb{Z}_{3} \times \mathbb{Z}_{3}$ is ruled out, and both $\mathbb{Z}_{2} \times \mathbb{Z}_{2}$ and $\mathbb{Z}_{2} \times \mathbb{Z}_{4}$ imply Type 1. As for the other groups we have $g \geq 1+(k / 2)-(k / 2 a)-a$, $k>4,2 \leq a \leq \sqrt{k}$. Write $f(X)=1+(k / X)-(k / 2 X)-X$. The minimum values of $f(X)$ in the range $2 \leq X \leq \sqrt{k}$ are either $f(2)=(k / 4)-1$ or $f(\sqrt{k})=1+(k / 2)-(3 \sqrt{k} / 2)$. Suppose $k \geq 16$. One has $f(2) \leq f(\sqrt{k})$, so that $g \geq f(a) \geq f(2)$. Hence $k \leq 4 g+4$, and either $S$ belongs to Type 1 or it belongs to Type 2 with $g=3$ and $G$ equal to $\mathbb{Z}_{2} \times \mathbb{Z}_{8}$ or $\mathbb{Z}_{4} \times \mathbb{Z}_{4}$. Now assume $4<k<16$. Since $k$ is a multiple of 4 and the case $G=\mathbb{Z}_{2} \times \mathbb{Z}_{4}$ has already been considered, we are reduced to $k=12$, that is to say, $G=\mathbb{Z}_{2} \times \mathbb{Z}_{6}$. In this case, $S$ must belong to Types 1 or 3 . Summing up, we can provisionally say that either $S$ is of Type 1 or one of the following holds:

\begin{tabular}{|c|c|c|}
\hline Type & $G$ & $g$ \\
\hline 2 & $\mathbb{Z}_{8}$ & 2 \\
2 & $\mathbb{Z}_{2} \times \mathbb{Z}_{8}$ & 3 \\
2 & $\mathbb{Z}_{4} \times \mathbb{Z}_{4}$ & 3 \\
3 & $\mathbb{Z}_{2} \times \mathbb{Z}_{6}$ & 2 \\
\hline
\end{tabular}

Claim 1. If $S$ is of Type 1 then $g=2$ or 3 .

Proof of Claim 1. Pick an element $\tau \in G$ fixing a given point $P \in D$, and write $A:=D /(\tau), H:=G /(\tau)$. The projection $\gamma: D \rightarrow D / G$ factors through

$$
D \stackrel{\alpha}{\longrightarrow} D /(\tau)=A \stackrel{\beta}{\longrightarrow} D / G \simeq \mathbb{P}^{1}
$$

where $\alpha$ and $\beta$ are Galois morphisms with groups $(\tau) \simeq \mathbb{Z}_{2}$ and $H$ respectively. Since $\gamma$ is ramified of order 2 at $P$, it follows that $\beta$ is unramified at $\alpha(P)$, and then $\beta^{-1} \beta \alpha(P)$ consists of $2 g-2$ distinct points, all of them branch points of $\alpha$ with branching order 2. Hurwitz's formula on $\alpha$ yields $2 g-2 \geq 2(2 g(A)-2)+2 g-2$, and thus $g(A)=0$ or 1 . Observe that the branching orders of $\beta$ are at most 2. If $g(A)=0$ then Proposition 2.5 applies and one gets $H \simeq \mathbb{Z}_{2}$ or $\mathbb{Z}_{2} \times \mathbb{Z}_{2}$, so that $G=\mathbb{Z}_{4}, \mathbb{Z}_{2} \times \mathbb{Z}_{2}$, or $\mathbb{Z}_{2} \times \mathbb{Z}_{4}$, and $g=2$ or 3. Assume $g(A)=1$, and choose $\sigma \in H$ fixing some point of $A$. 
The morphism $\beta$ factors through Galois maps

$$
A \rightarrow A /(\sigma) \simeq \mathbb{P}^{1} \rightarrow A / H \simeq \mathbb{P}^{1} .
$$

Applying again Proposition 2.5 to the second arrow we get that $H /(\sigma)$ is either $0, \mathbb{Z}_{2}$ or $\mathbb{Z}_{2} \times \mathbb{Z}_{2}$. Hence $g=2,3$ or 5 . But the case $g=5$ is ruled out with the following argument. The possibilities for $G$, in case $g=5$, are $\mathbb{Z}_{16}, \mathbb{Z}_{2} \times \mathbb{Z}_{8}$ or $\mathbb{Z}_{4} \times \mathbb{Z}_{4}$, and thus $G$ contains at most 4 elements of order 2. If $\tau \in G$ is one such element then $g(D /(\tau))=1$, as we know by the arguments above, so that $D \rightarrow$ $D /(\tau)$ has 8 ramification points. Equivalently, 8 points of $D$ are left fixed by $\tau$. This computation yields a maximum of 32 ramification points of $\gamma: D \rightarrow D / G$, while we know that $\gamma$ should have exactly 40 ramification points if $g=5$, absurd.

Claim 2. Type 1 and $G=\mathbb{Z}_{2} \times \mathbb{Z}_{4}$ is not possible.

Proof of Claim 2. $G$ has only three elements $\tau_{1}, \tau_{2}, \tau_{3}$ of order 2. Applying Hurwitz's formula to $D \rightarrow D /\left(\tau_{i}\right)$ one sees that $\tau_{i}$ is fixing $8-4 g_{i}$ points on $D$, where $g_{i}$ denotes the genus of $D /\left(\tau_{i}\right)$. Since $D \rightarrow D / G$ has 20 ramification points, the only possibility (up to order) is to have $g_{1}=g_{2}=0, g_{3}=1$. But this contradicts the fact that a hyperelliptic involution is uniquely determined.

Claim 3. Type 1 and $G$ cyclic is impossible.

Proof of Claim 3. It is enough to consider the cases $G=\mathbb{Z}_{4}$ and $G=\mathbb{Z}_{8}$. In both cases there is only one point of order 2 , which fixes a maximum of 6 or 8 points of $D$ respectively. But this is not enough to account for all the ramification points of $\gamma: D \rightarrow D / G$, whose number we know a priori (10 and 20 respectively).

Claim 4. Type 2 and $G=\mathbb{Z}_{8}$ is impossible.

Proof of Claim 4. The branching orders of $\gamma: D \rightarrow D / G$ are $(4,4$, 4), so that there exist 6 ramification points on $D$. Let $\tau \in G$ be an element of order 4 fixing a point of $D$, and factor $\gamma$ as

$$
D \stackrel{\alpha}{\rightarrow} D /(\tau)=A \rightarrow D / G \simeq \mathbb{P}^{1} .
$$

Hurwitz's formula for $\alpha$ yields $2=2 g-2=4(2 g(A)-2)+r$, with $r \geq 3$ being the order of the ramification divisor. Thus $g(A)=0$ and $r=10$, which implies that $\tau$ cannot fix more than 2 points of $D$. 
Since $\mathbb{Z}_{8}$ has only 2 elements of order 4 we would obtain a maximum of 4 ramification points for $\gamma$, while there should be exactly 6 with our hypothesis, a contradiction.

Claim 5. Type 2 and $G=\mathbb{Z}_{2} \times \mathbb{Z}_{8}$ is not possible.

Proof of Claim 5. Let $\tau=(1,0) \in \mathbb{Z}_{2} \times \mathbb{Z}_{8}$, so that $G /(\tau) \simeq \mathbb{Z}_{8}$, and decompose $\gamma: D \rightarrow D / G$ as

$$
D \stackrel{\alpha}{\longrightarrow} D /(\tau)=A \stackrel{\beta}{\longrightarrow} D / G \simeq \mathbb{P}^{1} .
$$

One has $g(A) \leq 2$. The case $g(A)=2$ is ruled out by Claim 4 , since then $\alpha$ would be étale and $\beta$ would have branching orders $(4,4,4)$. Proposition 2.5 eliminates the possibility $g(A)=0$, so it suffices to consider $g(A)=1$. Now we have that $G /(\tau) \simeq \mathbb{Z}_{8}$ acts on the elliptic curve $A$ with ramification. By the structure of the automorphisms of elliptic curves and the fact that $G /(\tau)$ is abelian we have that $G /(\tau)$ is a direct product of $\mathbb{Z}_{m}$ and $\mathbb{Z}_{n}$ where $\mathbb{Z}_{m}$ is the subgroup of $G /(\tau)$ consisting of translations of $A$ and $\mathbb{Z}_{n}$ are group-automorphisms of $A$, so that $2 \leq n \leq 6$. But this is obviously impossible.

Combining these five claims with the last statement preceding Claim 1 ends the proof of our theorem.

Next we are going to give an explicit construction of some elliptic surfaces with a genus 2 ample divisor. In fact, this method yields all surfaces which belong to Type 1 of Theorem 2.2 .

EXAmple 2.6. Let $C_{1}, C_{2}$, be two copies of $\mathbb{P}^{1}, C_{3}$ an elliptic curve, and let $\rho: C_{2} \rightarrow C_{1}, \sigma: C_{3} \rightarrow C_{1}$ be double covers whose branch loci have a single point $Q \in C_{1}$ in common. Denote by $D$ the normalization of $C_{2} \times_{C_{1}} C_{3}$. We claim that $D$ is a smooth irreducible curve of genus 2 . On the one hand, both maps $D \rightarrow C_{2}, D \rightarrow C_{3}$ have degree 2 , so that if $D$ is disconnected then it consists of two connected components, each one simultaneously isomorphic to $C_{2}$ and $C_{3}$, absurd because $g\left(C_{2}\right) \neq g\left(C_{3}\right)$. Moreover, since smoothness of a morphism is preserved by base change one sees that $C_{2} \times{ }_{C_{1}} C_{3}$ is non-singular away from the fibre over $Q \in C_{1}$. Analytically, the singularity over $Q$ looks like $\left\{(x, y) \in \mathbb{C}^{2} \mid x^{2}-y^{2}=0\right\}$, and thus the fibre of $D$ over $Q$ consists of two distinct points. Therefore, $D \rightarrow C_{3}$ is a degree 2 map with two branch points, which implies $g(D)=2$. We can consider that $\mathbb{Z}_{2}$ is acting both on $C_{2}$ and $C_{3}$ yielding $C_{1}$ 
as a quotient. Then $(\alpha, \beta) \in \mathbb{Z}_{2} \times \mathbb{Z}_{2}$ acts on $\left(P_{2}, P_{3}\right) \in C_{2} \times_{C_{1}} C_{3}$ as $(\alpha, \beta)\left(P_{2}, P_{3}\right)=\left(\alpha P_{2}, \beta P_{3}\right)$. This action can be lifted to $D$, and $D /\left(\mathbb{Z}_{2} \times \mathbb{Z}_{2}\right)=C_{1}$.

Write $G:=\mathbb{Z}_{2} \times \mathbb{Z}_{2}$ and choose any elliptic curve $E$. We can make $G$ act faithfully on $E$ by translations. Let $S$ stand for the quotient $(D \times E) / G$, where $G$ is acting on $D \times E$ componentwise. We will call $E_{0}, D_{0}$ the general fibres of the two natural projections $\Phi: S \rightarrow$ $(D / G) \simeq \mathbb{P}^{1}, \Psi: S \rightarrow(E / G)$ respectively. Obviously $D_{0} E_{0}=4$.

As in the proof of Theorem 2.2 one sees that $D_{0}, E_{0}$ form a basis of $H^{2}(S, \mathbb{Q}), K_{S} \equiv(1 / 2) E_{0}$. Similarly one gets $\operatorname{dim} H^{1}(S, \mathbb{Q})=2$, which combined with $K_{S}^{2}=0$ and Noether's formula yields $\chi \mathscr{O}_{S}=0$, and thus $p_{g}(S)=0$. Inasmuch as all singular fibres of the elliptic fibration $\Phi$ have multiplicity 2, Proposition 1.4 says that $(1 / 2) E_{0}$ is not divisible by any integer greater than 1 . Now one can quote Poincaré duality as in ([8], p. 53) in order to get a divisor $L$ such that $(1 / 2) E_{0} \cdot L=1$. We have $L \equiv(1 / 2) D_{0}+k E_{0}$, for some $k \in \mathbb{Q}$, and $L^{2}+L K_{S}=4 k+1$. But $4 k+1$ must be an even integer because of the adjunction formula. It follows that by adding an integral multiple of $E_{0}$ to $L$ we may assume $L \equiv(1 / 2) D_{0}+(1 / 4) E_{0}$. Now we are done: it suffices to check $g(L)=2$ and to notice that $L$ is an ample divisor by reason of Nakai's criterion. Observe that $S$ belongs to Type 1 of Theorem 2.2.

\section{REFERENCES}

[1] W. Barth, C. Peters, and A. van de Ven, Compact Complex Surfaces, Springer, Berlin-Heidelberg-New York, 1984.

[2] A. Beauville, Surface algébriques complexes, Astérisque, 54 (1978).

[3] M. Beltrametti, A. Lanteri, and M. Palleschi, Algebraic surfaces containing an ample divisor of arithmetic genus two, Arkiv för Mat., 25 (1987), 189-210.

[4] M. Beltrametti and A. J. Sommese, On generically polarized Gorenstein surfaces of sectional genus two, J. Reine Angew. Math., 386 (1988), 172-186.

[5] H. M. Farkas and I. Kra, Riemann Surfaces, Grad. Texts in Math., 71, Springer, Berlin-Heidelberg-New York, 1980.

[6] T. Fujita, On polarized manifolds of sectional genus two, Proc. Japan Acad., 62 (1986), 69-72.

[7] _ Classification of polarized manifolds of sectional genus two, in "Algebraic Geometry and Commutative Algebra", in honor of Masayoshi Nagata; 1988, 73-93, Kinokuniya, Tokyo.

[8] P. Griffiths and J. Harris, Principles of Algebraic Geometry, John Wiley \& Sons, New York, 1978.

[9] W. J. Harvey, Cyclic groups of automorphisms of a compact Riemann surface, Quart. J. Math. Oxford, 17 (1966), 86-97.

[10] T. Katsura and K. Ueno, On ellipitic surfaces in characteristic p, Math. Ann., 272 (1985), 291-330. 
[11] C. Maclachlan, Abelian groups of automorphisms of compact Riemann surfaces, Proc. London Math. Soc., 15 (1965), 699-712.

[12] F. Serrano, The Picard group of a quasi-bundle, eibpreprint.

[13] _ On isotrivial fibrations, preprint

[14] I. R. Shafarevich, et al., Algebraic Surfaces, Proc. Steklov Inst. Math., 75 (1965), Amer. Math. Soc. Translations, Providence, R.I. (1967).

Received July 12, 1990 and in revised form August 10, 1990.

Departament d’Àlgebra i Geometria

Facultat de MATEMÀtiques

UNIVERSITAT DE BARCELONA

Gran Via 585, 08007 Barcelona, Spain 



\title{
PACIFIC JOURNAL OF MATHEMATICS EDITORS
}

\author{
V. S. VARADARAJAN \\ (Managing Editor) \\ University of California \\ Los Angeles, CA 90024-1555-05 \\ Herbert Clemens \\ University of Utah \\ Salt Lake City, UT 84112 \\ F. Michael Christ \\ University of California \\ Los Angeles, CA 90024-1555 \\ THOMAS ENRIGHT \\ University of California, San Diego \\ La Jolla, CA 92093
}

Nicholas ERcolani

University of Arizona

Tucson, AZ 85721

R. FINN

Stanford University

Stanford, CA 94305

VAUGHAN F. R. JoNeS

University of California

Berkeley, CA 94720

C. C. MOORE

University of California

Berkeley, CA 94720

MARTIN SchaRLEMANN

University of California

Santa Barbara, CA 93106

HAROLd StaRK

University of California, San Diego

La Jolla, CA 92093

SteVen Kerckhoff

Stanford University

Stanford, CA 94305

\section{ASSOCIATE EDITORS}

\begin{tabular}{|c|c|c|c|c|}
\hline R. ARENS & $\begin{array}{l}\text { E. F. BECKENBACH } \\
(1906-1982)\end{array}$ & Neumann & $\begin{array}{c}\text { F. WoLF } \\
(1904-1989)\end{array}$ & K. YosHIDA \\
\hline \multicolumn{5}{|c|}{ SUPPORTING INSTITUTIONS } \\
\hline \multicolumn{2}{|c|}{ UNIVERSITY OF ARIZONA } & \multicolumn{3}{|c|}{ UNIVERSITY OF OREGON } \\
\hline \multicolumn{2}{|c|}{ UNIVERSITY OF BRITISH COLUMBIA } & \multicolumn{3}{|c|}{ UNIVERSITY OF SOUTHERN CALIFORNI } \\
\hline \multicolumn{2}{|c|}{ CALIFORNIA INSTITUTE OF TECHNOLOGY } & \multirow{2}{*}{\multicolumn{3}{|c|}{ STANFORD UNIVERSITY }} \\
\hline \multicolumn{2}{|c|}{ UNIVERSITY OF CALIFORNIA } & & & UNIVERSITY OF HAWAII \\
\hline \multicolumn{2}{|c|}{ MONTANA STATE UNIVERSITY } & \multicolumn{3}{|c|}{ UNIVERSITY OF TOKYO } \\
\hline \multicolumn{2}{|c|}{ UNIVERSITY OF NEVADA, RENO } & \multicolumn{3}{|c|}{ UNIVERSITY OF UTAH } \\
\hline \multicolumn{2}{|c|}{ NEW MEXICO STATE UNIVERSITY } & \multicolumn{3}{|c|}{ WASHINGTON STATE UNIVERSITY } \\
\hline \multicolumn{2}{|c|}{ OREGON STATE UNIVERSITY } & UNIVER: & OF WASHINC & \\
\hline
\end{tabular}




\section{Pacific Journal of Mathematics}

Vol. 152, No. $1 \quad$ January, 1992

B. V. Rajarama Bhat, On a characterization of velocity maps in the space of observables .......................................

John David Brillhart, Note on the discriminant of certain cyclotomic period

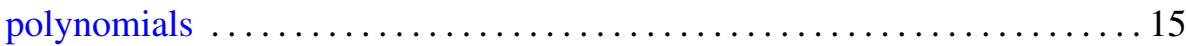

Steven R. Costenoble and Stefan Waner, The equivariant Thom

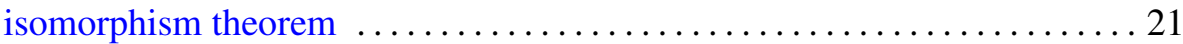

Karl Heinz Dovermann and Dong Youp Suh, Smith equivalence for finite

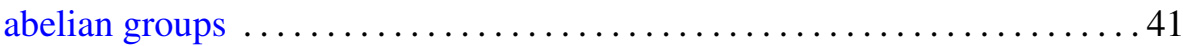

Klaus-Jochen Engel, On singular perturbations of second order Cauchy

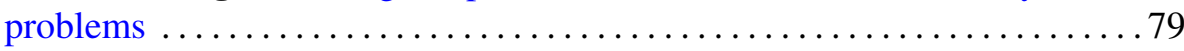

Angel Ferrandez and Pascual Lucas, On surfaces in the 3-dimensional

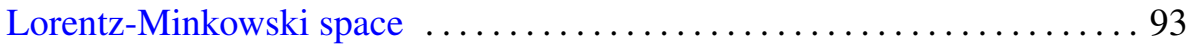

Brian Hartley, A general Brauer-Fowler theorem and centralizers in locally

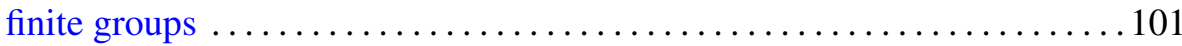

Joel Hass, Intersections of least area surfaces .................... 119

Edward Kissin, Indices of unbounded derivations of $C^{*}$-algebras $\ldots \ldots \ldots 125$

Erhard Luft and Denis Karmen Sjerve, On regular coverings of

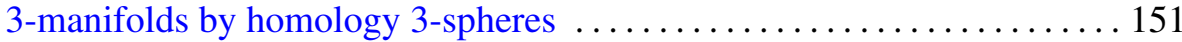

Daniel Pecker, Sur la projection de variétés algébriques réelles .......... 165

Claus Scheiderer, Some remarks on orderings under finite field

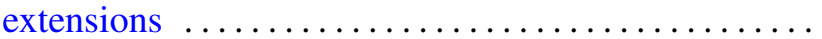

Fernando Serrano, Elliptic surfaces with an ample divisor of genus two 